\title{
Successful Refractory of Acute Myocardial Infarction-induced Electrical Storm by Defibrillations and Amiodarone: A Case Report
}

Huanhuan Guo

Huazhong University of Science and Technology

Quan Gan (D Ganquan0116@hotmail.com )

Huazhong University of Science and Technology

\section{Case report}

Keywords: Electrical Storm, Ventricular Tachycardia, Defibrillation, Case Report

Posted Date: July 7th, 2020

DOI: https://doi.org/10.21203/rs.3.rs-39555/v1

License: (c) (1) This work is licensed under a Creative Commons Attribution 4.0 International License.

Read Full License 


\section{Abstract}

Background: Acute myocardial infarction (AMI) often complicated with multiple arrhythmias, especially ventricular arrhythmias, including sustained ventricular tachycardia (SVT) and ventricular fibrillation (VF) are often presages progressive heart failure in 48 hours. The present study reports a case of electrical storm (ES) occurring in an 84-year-old woman with acute myocardial infarction (AMI).

Case presentation: With the defibrillations or amiodarone, the recurrence of ventricular tachycardia inhibited and the electrocardiographic pattern normalized.

Conclusions: The results suggest that defibrillations and amiodarone may be able to improve the survival rate of patients with ES with $\mathrm{AMI}$ and would be considered as an alternative treatment for implantable cardioverter defibrillator (ICD) and invasive catheter ablation in the management of cardiac ES.

\section{Background}

Electrical storm (ES) is a distinct medical emergency characterized by three or more discrete episodes of ventricular arrhythmia (ventricular tachycardia, VT/ventricular fibrillation, VF) within 24 hours, or incessant ventricular arrhythmia for more than 12 hours, requiring the intervention of the defibrillator (antitachycardia pacing or shock) ${ }^{12}$, and portends a significant increase in mortality risk and often presages progressive heart failure ${ }^{34}$. Lower left ventricular ejection fraction, implantation of a secondary prevention implantable cardioverter defibrillator (ICD), treatment with class I anti-arrhythmic agents, and monomorphic ventricular tachycardia (VT) as the underlying arrhythmia associated with a higher incidence of ES ${ }^{5}$. The pathophysiological mechanisms which underlie ventricular arrhythmia storm were not well characterized. It is likely that there is a complex interplay between enhanced sympathetic tone ${ }^{6}$, calcium-related signaling abnormalities, dysregulation of protein phosphorylation and a susceptible arrhythmogenic substrate ${ }^{7}$. Patients with ES are more likely to have advanced cardiac disease.

The management of ES requires complex multi-disciplinary care, including ICD re-programming, haemodynamic support, the use of antiarrhythmic medications, identification and reversal of precipitating factors, and consideration of catheter ablation and neuraxial modulation. Radiofrequency catheter ablation (CA) is emerging as the standard-of-care in patients with electrical storm refractory to optimal medical management.

We report a unique case of acute myocardial infarction-induced electrical storm with low-output heart failure. Although the patient showed vital signs consistent with shock during the procedure, she was successfully rhythm-controlled with electrical defibrillation and amiodarone.

\section{Case Presentation}

In this case report, we describe a case of an 84-year-old woman, was brought to the emergency department (ED) by ambulance with symptoms of occasional chest tightness occurring over the previous 
4 years, and recurrent over the preceding 2 days, sudden chest tightness persisting for 1 hour and without relief. And the patient was shifted to the Medical Intensive Care Unit (MICU). On admission, she had AMI for worsening heart failure, with previous medical history significant for more than 20 years of hypertension (grade 3 , very high-risk group), dyslipidemia, coronary artery disease (CAD) and twice percutaneous coronary intervention $(\mathrm{PCl})$ and two stents placement surgery history. And she has a longterm oral thiazide diuretics history for chronic renal insufficiency (CRI) elevated creatinine and oliguria. And she was suffering chronic constipation (CC) in the past 1 year, and had a long-term oral rhubarb soda tablets. And she had a positive history for nitroglycerin, penicillin, and cephalosporin. On physical examination, low heart sound and pulmonary wet rales were heard on auscultation, ambiguous and with a pained expression. Her electrocardiogram (ECG) demonstrated monomorphic VT, 168 beats per minute (bpm) and irregular, 37 breaths per minute (bpm), blood pressure (BP) was $80 / 60 \mathrm{mmHg}$, and blood oxygen saturation $\left(\mathrm{SpO}_{2}\right)$ was $96-99 \%$.

Defibrillation using 200J was immediately performed with restoration of sinus rhythm (70 bpm), with an elevated blood pressure present, and the patient appeared drowsy, with a Glasgow Coma Scale (GCS) score of 13 (E3V5M5). Then she was received clopidogrel and aspirin for oral administration, low molecular weight heparin (LMWH) subcutaneous injection, dopamine infusion to maintain blood pressure, lansoprazole to inhibit of gastric acid secretion, and the ECG monitor displayed, BP 117/75 mmHg, HR 64 bpm (sinus rhythm), R 19 bpm.

She was assessed as Killip IV-grade. Her troponin I level was elevated at $11.871 \mathrm{ng} / \mathrm{ml}$ (normal 0$0.04 \mathrm{ng} / \mathrm{ml}$ ), and brain natriuretic peptide (BNP) concentration was $1348.8 \mathrm{pg} / \mathrm{ml}$ (normal $<100 \mathrm{pg} / \mathrm{ml}$ ). A 12-lead ECG revealed sine-wave pattern and ST elevation with features of low cardiac output. On transthoracic echocardiography, the left atrium (LA) and left ventricle (LV) diameter was $35 \mathrm{~mm}$ and $65 \mathrm{~mm}$, respectively. The ventricular septum was $60 \mathrm{~mm}$, and the posterior wall of the left ventricle was $60 \mathrm{~mm}$, and the activity of them was reversed and the wall motion exhibited globally severe hypokinesis with an LV ejection fraction (LVEF) of $13 \%$.

The patient was monitored in the MICU while experiencing unconsciousness, shallow breathing, hypotension, and hypoperfusion of end organs (cool extremities, low urine output and heart rate of 50$75 \mathrm{bpm}$ ) and supported with dopamine, the urine volume was $1299 \mathrm{ml}$ within the first 24 hours.

After defibrillation, a 12-lead ECG depicted normal sinus rhythm, premature ventricular contractions, firstdegree atrioventricular block, ST-T segment changed in leads $\mathrm{Q}, \mathrm{Q}, \mathrm{Q}, \mathrm{aVL}, \mathrm{aVF}, \mathrm{V} 4$ through V9, T-wave changed in leads V2-V4, suggested old myocardial infarction.

The results of complete blood count indicated a rise in leukocyte and neutrophil counts, and serum concentrations of electrolytes were normal, creatinine $387 \mathrm{umol} / \mathrm{L}(45-84 \mathrm{umol} / \mathrm{L})$, and liver function tests were ALT $1190 \mathrm{U} / \mathrm{L}$ (0-40 U/L), AST $1578.4 \mathrm{U} / \mathrm{L}$ (0-35 U/L), TBA 22.2umol/L (0.5-10umol/L), LDH $1776 \mathrm{U} / \mathrm{L}(120-250 \mathrm{U} / \mathrm{L})$. Coagulation function showed D-DI 26.3ug/mL (< 0.5ug/mL). 
On hospital day 2, the patient developed a recurrent wide complex QRS that transitioned into sustained monomorphic ventricular tachycardia (SMVT), requiring electrical defibrillation. After defibrillation, the patient's QRS interval normalized, SMVT occurred again, just 6 minutes after defibrillation, then after 3 times of defibrillation, antiarrhythmic treatment with amiodarone was initiated immediately, we administered an intravenous infusion of $150 \mathrm{mg}$ amiodarone, followed by a continuous infusion $(1 \mathrm{mg} / \mathrm{min}$ ) as a maintenance volume for 6 hours. But this also failed to terminate the occurrence of arrhythmia, SMVT occurred repeatedly, with a total of 9 ventricular arrhythmias rapidly degenerating to ventricular tachycardia with hypotension within 8 hours, fortunately, electrical defibrillation could restore the sinus rhythm, and the interval was extended with the amiodarone maintenance. Over the next 12 hours, each SMVT could be converted to sinus rhythm through one or two doses of intravenous amiodarone (150 mg each, intravenously) without defibrillation, and since had no documented episodes of ventricular tachycardia. Her non-invasive blood pressure (NBP) varied between $65 / 42 \mathrm{mmHg}$ and $151 / 90 \mathrm{mmHg}$, and his pulse ranged from 99 to $182 \mathrm{bpm}$, with a stable respiratory rate from 12 to $20 \mathrm{bpm}$.

A post-cardioversion ECG confirmed sinus rhythm. She was continuous amiodarone with $0.5 \mathrm{mg} / \mathrm{min}$ infusion for 2 days after first 6 hours with $1 \mathrm{mg} / \mathrm{min}$. After defibrillation and amiodarone infusion, the ECG changes began to normalize gradually, contractility of the heart also improved. On the next days in hospital, her mental status returned to her baseline, and her GCS score was 15. And several daily ECGs during hospitalization depicted normal sinus rhythm. The patient was discharged her 10th day in the hospital with clinically stable and medically asymptomatic.

The patient remained free from sustained ventricular arrhythmia during the six-month follow-up.

\section{Discussion}

Acute myocardial infarction (AMI) often complicated with multiple arrhythmias, especially ventricular arrhythmias, including sustained ventricular tachycardia (SVT) and ventricular fibrillation (VF) are often presages progressive heart failure in 48 hours. Previous studies have confirmed that the occurrence of SVT and VF is associated with myocardial infarct size and LVEF, and the risk of cardiogenic shock and inhospital death is significantly higher ${ }^{8-10}$. And it is important to identify SVT/VF high-risk groups and take effective protective measures to improve the prognosis of patients with AMI early. Practice has shown that early and immediate defibrillation is the most effective treatment.

According to the guidelines of American College of Cardiology Foundation (ACCF)/American Heart Association (AHA) and European Society of Cardiology (ESC), for patients with hemodynamic instability, emergency PCl-based revascularization should be performed as soon as possible ${ }^{11}{ }^{12}$, and the time window of emergency $\mathrm{PCl}$ can be relaxed accordingly. The ACC/AHA have pointed out the treatment guidelines for ST-segment elevation myocardial infarction (STEMI) that patients with AMI complicated with ventricular tachycardia are preferred for intravenous amiodarone in hemodynamically stable conditions ${ }^{13}$. Otherwise, the cardioversion is performed first, followed by amiodarone. 
We present a case of a patient with recurrent unstable SMVT, who was successfully cardioverted with defibrillations or amiodarone. SMVT can result in hypoperfusion or devolve into more dangerous rhythms such as VF.

Current ventricular tachycardia management in patients with ischemic cardiomyopathy (ICM) includes optimal medical therapy, ICD device therapy, and antiarrhythmic medications. Sustained ventricular tachyarrhythmia continues to be a significant cause of morbidity and mortality in patients with ischemic cardiomyopathy (ICM) ${ }^{14}$. The placement of ICD is proven to reduce the rates of sudden death and mortality in patients with ICM and reduced ejection fraction ${ }^{15}$. However, the ICD has no effect on the incidence or recurrence of the events. Recurrent ventricular tachyarrhythmias can lead to recurrent shocks and re-hospitalizations, and were proven to be associated with worse overall outcomes ${ }^{16-20}$. Catheter ablation aims to prevent recurrence of VT, thus in theory, reducing recurrent ICD shocks and subsequently the need for long-term use of potentially toxic antiarrhythmic agents. Worldwide, invasive catheter ablation is increasingly performed to treat ventricular tachycardia ${ }^{21}$. The procedure is largely effective in the absence of ventricular scar (idiopathic ventricular tachycardia). However, catheter ablation for cardiomyopathic ventricular tachycardia is associated with recurrence rates as high as $50 \%$ at 6 months $22-27$.

Amiodarone is commonly used in the treatment of ES. It exerts a predominant Class III effect by way of potassium channel blockade and resultant prolongation of the refractory period in cardiac myocytes. It also exhibits Class I (sodium channel inhibition), Class II (L-type calcium channel blockade) and Class IV (sympathetic blockade) effects, most notably when administered intravenously. Amiodarone has been demonstrated to successfully terminate ventricular arrhythmias in approximately $40 \%$ of patients when used in isolation, and is useful in preventing long-term recurrence ${ }^{28}{ }^{29}$. Amiodarone plus $\beta$-blockers significantly reduces the risk of recurrent ICD-shocks compared with $\beta$-blockers alone and sotalol alone 29 . In ES, amiodarone may reduce storm recurrences four-fold over 2-year follow-up ${ }^{30}$. In addition to its special electrophysiological effects, amiodarone has the function of dilation coronary arteries, increasing blood flow, dilating peripheral arteries, reducing peripheral resistance, myocardial ischemia and myocardial oxygen consumption, especially in the ischemic arrhythmia, on the other hand, intravenous injection can lower blood pressure and even cause fatal cardiogenic shock, but has no significant effect on cardiac output. Amiodarone has no or only mild negative inotropic effects and has a good effect on arrhythmias complicated with heart failure. Studies have reported that the intravenous of amiodarone in the acute phase can make ICD storms stable in a short period of time. Unfortunately, amiodarone is not a benign medication, and the long-term use of amiodarone is often limited by its associated toxicities, including liver dysfunction, hypo- and hyperthyroidism, pulmonary fibrosis and, most notably, proarrhythmic effects, including precipitating VF and torsades de pointes. Furthermore, chronic amiodarone therapy has been associated with increased defibrillation thresholds ${ }^{31}$. A recent meta-analysis has suggested amiodarone may be associated with increased mortality ${ }^{32}$. 
AHA guidelines recommend antiarrhythmic treatment with amiodarone in patients with monomorphic VT for which electrical cardioversion has been unsuccessful. And most of the deaths were attributed to congestive heart failure or noncardiac causes, with few deaths from arrhythmia. In this case, she is an elderly woman with multiple organ dysfunctions, and we have no medical conditions to undergo emergency PCl. And the patient's quality of life was acceptable during the period of 6-month follow-up with our active treatment.

\section{Conclusion}

We describe a case of successful cardioversion by electrical defibrillation and amiodarone in a patient with recurrent ventricular tachycardia storm. Although further research is needed to assess the safety of the bolus infusion of amiodarone, that may worsen hypotension in already unstable patients, and the long-term efficacy, especially in elderly patients with pulmonary insufficiency. Consideration may be given to using this method in patients with unstable SMVT when medical conditions do not allow coronary stent placement in case, demonstrated safety and effectiveness of this technique could reduce the damage of invasive operations.

\section{Declarations}

\section{Ethics approval and consent to participate}

The patient agreed to participate in the study.

\section{Consent for publication}

All the authors approved the final paper.

\section{Availability of data and materials}

Not applicable.

\section{Competing interests}

There are no conflicts of interest.

\section{Funding}

None.

\section{Authors' contributions}

The first author collected the case data and wrote the manuscript, the corresponding author made constructive comments and revised the paper, and both agree to be accountable for all aspects of the manuscript. 
Acknowledgements

Not applicable.

\section{References}

1. Pedersen CT, Kay GN, Kalman J, et al. EHRA/HRS/APHRS expert consensus on ventricular arrhythmias. Heart rhythm. 2014;11(10):e166-96.

2. Geraghty L, Santangeli P, Tedrow UB, et al. Contemporary Management of Electrical Storm. Heart Lung Circ. 2019;28(1):123-33.

3. Guerra F, Shkoza M, Scappini L, et al. Role of electrical storm as a mortality and morbidity risk factor and its clinical predictors: a meta-analysis. Europace: European pacing, arrhythmias, and cardiac electrophysiology : journal of the working groups on cardiac pacing, arrhythmias, and cardiac cellular electrophysiology of the European Society of Cardiology 2014;16(3):347 - 53.

4. Noda T, Kurita T, Nitta T, et al. Significant impact of electrical storm on mortality in patients with structural heart disease and an implantable cardiac defibrillator. Int J Cardiol. 2018;255:85-91.

5. Vergara P, Tung R, Vaseghi M, et al. Successful ventricular tachycardia ablation in patients with electrical storm reduces recurrences and improves survival. Heart rhythm. 2018;15(1):48-55.

6. Vaseghi M, Gima J, Kanaan C, et al. Cardiac sympathetic denervation in patients with refractory ventricular arrhythmias or electrical storm: intermediate and long-term follow-up. Heart rhythm. 2014;11(3):360-6.

7. Tsuji $\mathrm{Y}$, Hojo $\mathrm{M}$, Voigt $\mathrm{N}$, et al. $\mathrm{Ca}(2+)$-related signaling and protein phosphorylation abnormalities play central roles in a new experimental model of electrical storm. Circulation. 2011;123(20):2192203.

8. Mehta RH, Starr AZ, Lopes RD, et al. Incidence of and outcomes associated with ventricular tachycardia or fibrillation in patients undergoing primary percutaneous coronary intervention. Jama. 2009;301(17):1779-89.

9. Incidence and Risk Factors of Ventricular Fibrillation. Before Primary Angioplasty in Patients With First ST-Elevation Myocardial Infarction: A Nationwide Study in Denmark. Journal of the American Heart Association 2015;4(7).

10. Al-Khatib SM, Stebbins AL, Califf RM, et al. Sustained ventricular arrhythmias and mortality among patients with acute myocardial infarction: results from the GUSTO-III trial. American heart journal. 2003;145(3):515-21.

11. O'Gara PT, Kushner FG, Ascheim DD, et al. 2013 ACCF/AHA guideline for the management of STelevation myocardial infarction: a report of the American College of Cardiology Foundation/American Heart Association Task Force on Practice Guidelines. J Am Coll Cardiol. 2013;61(4):e78-140.

12. Arslan F, Bongartz L, Ten Berg JM, et al. 2017 ESC guidelines for the management of acute myocardial infarction in patients presenting with ST-segment elevation: comments from the Dutch 
ACS working group. Netherlands heart journal: monthly journal of the Netherlands Society of Cardiology the Netherlands Heart Foundation. 2018;26(9):417-21.

13. Antman EM, Anbe DT, Armstrong PW, et al. ACC/AHA guidelines for the management of patients with ST-elevation myocardial infarction-executive summary: a report of the American College of Cardiology/American Heart Association Task Force on Practice Guidelines (Writing Committee to Revise the 1999 Guidelines for the Management of Patients With Acute Myocardial Infarction). Circulation. 2004;110(5):588-636.

14. Streitner F, Kuschyk J, Dietrich C, et al. Comparison of ventricular tachyarrhythmia characteristics in patients with idiopathic dilated or ischemic cardiomyopathy and defibrillators implanted for primary prevention. Clin Cardiol. 2011;34(10):604-9.

15. Moss AJ, Hall WJ, Cannom DS, et al. Improved survival with an implanted defibrillator in patients with coronary disease at high risk for ventricular arrhythmia. Multicenter Automatic Defibrillator Implantation Trial Investigators. N Engl J Med. 1996;335(26):1933-40.

16. Dunbar SB, Dougherty CM, Sears SF, et al. Educational and psychological interventions to improve outcomes for recipients of implantable cardioverter defibrillators and their families: a scientific statement from the American Heart Association. Circulation. 2012;126(17):2146-72.

17. Poole JE, Johnson GW, Hellkamp AS, et al. Prognostic importance of defibrillator shocks in patients with heart failure. N Engl J Med. 2008;359(10):1009-17.

18. Sweeney MO, Sherfesee L, DeGroot PJ, et al. Differences in effects of electrical therapy type for ventricular arrhythmias on mortality in implantable cardioverter-defibrillator patients. Heart Rhythm. 2010;7(3):353-60.

19. Kuck KH, Cappato R, Siebels J, et al. Randomized comparison of antiarrhythmic drug therapy with implantable defibrillators in patients resuscitated from cardiac arrest: the Cardiac Arrest Study Hamburg (CASH). Circulation. 2000;102(7):748-54.

20. Maskoun W, Saad M, Abualsuod A, et al. Outcome of catheter ablation for ventricular tachycardia in patients with ischemic cardiomyopathy: A systematic review and meta-analysis of randomized clinical trials. Int J Cardiol. 2018;267:107-13.

21. Priori SG, Blomstrom-Lundqvist C, Mazzanti A, et al. 2015 ESC Guidelines for the management of patients with ventricular arrhythmias and the prevention of sudden cardiac death: The Task Force for the Management of Patients with Ventricular Arrhythmias and the Prevention of Sudden Cardiac Death of the European Society of Cardiology (ESC)Endorsed by: Association for European Paediatric and Congenital Cardiology (AEPC). Europace. 2015;17(11):1601-87.

22. Stevenson WG, Wilber DJ, Natale A, et al. Irrigated radiofrequency catheter ablation guided by electroanatomic mapping for recurrent ventricular tachycardia after myocardial infarction: the multicenter thermocool ventricular tachycardia ablation trial. Circulation. 2008;118(25):2773-82.

23. Tokuda M, Kojodjojo P, Tung $S$, et al. Acute failure of catheter ablation for ventricular tachycardia due to structural heart disease: causes and significance. J Am Heart Assoc. 2013;2(3):e000072. 
24. Dinov B, Fiedler L, Schonbauer R, et al. Outcomes in catheter ablation of ventricular tachycardia in dilated nonischemic cardiomyopathy compared with ischemic cardiomyopathy: results from the Prospective Heart Centre of Leipzig VT (HELP-VT). Study Circulation. 2014;129(7):728-36.

25. Marchlinski FE, Haffajee Cl, Beshai JF, et al. Long-Term Success of Irrigated Radiofrequency Catheter Ablation of Sustained Ventricular Tachycardia: Post-Approval THERMOCOOL VT Trial. J Am Coll Cardiol. 2016;67(6):674-83.

26. Tung R, Vaseghi M, Frankel DS, et al. Freedom from recurrent ventricular tachycardia after catheter ablation is associated with improved survival in patients with structural heart disease: An International VT Ablation Center Collaborative Group study. Heart Rhythm. 2015;12(9):1997-2007.

27. Santangeli P, Frankel DS, Tung R, et al. Early Mortality After Catheter Ablation of Ventricular Tachycardia in Patients With Structural Heart Disease. J Am Coll Cardiol. 2017;69(17):2105-15.

28. Effect of prophylactic amiodarone on mortality after. acute myocardial infarction and in congestive heart failure: meta-analysis of individual data from 6500 patients in randomised trials. Amiodarone Trials Meta-Analysis Investigators. Lancet. 1997;350(9089):1417-24.

29. Connolly SJ, Dorian P, Roberts RS, et al. Comparison of beta-blockers, amiodarone plus beta-blockers, or sotalol for prevention of shocks from implantable cardioverter defibrillators: the OPTIC Study: a randomized trial. Jama. 2006;295(2):165-71.

30. Greene M, Newman D, Geist M, et al. Is electrical storm in ICD patients the sign of a dying heart? Outcome of patients with clusters of ventricular tachyarrhythmias. Europace: European pacing, arrhythmias, and cardiac electrophysiology : journal of the working groups on cardiac pacing, arrhythmias, and cardiac cellular electrophysiology of the European Society of Cardiology 2000;2(3):263-9.

31. Jung W, Manz M, Pizzulli $L$, et al. Effects of chronic amiodarone therapy on defibrillation threshold. The American journal of cardiology. 1992;70(11):1023-7.

32. Santangeli P, Muser D, Maeda S, et al. Comparative effectiveness of antiarrhythmic drugs and catheter ablation for the prevention of recurrent ventricular tachycardia in patients with implantable cardioverter-defibrillators: A systematic review and meta-analysis of randomized controlled trials. Heart rhythm. 2016;13(7):1552-9. 